\title{
SOBRE LA CONNOTACIÓN
}

\author{
MASSIMO A. BONFANTINI \\ (Universidad de Nápoles)
}

\begin{abstract}
A) Denotación, connotación, evocación: interpretante inmediato, interpretación solicitada, semiosis ilimitada
\end{abstract}

I. Dicho sin diplomacia, creo que la orientación propuesta por Hjelmslev para los estudios de lingüistica y semiótica, tomada en sentido global, resulta más interesante para la historia de la cultura que para la futura producción científica. Y ello no sólo porque sus Fundamentos de la teoria del lenguaje están plenamente inscritos en la prospectiva de la "semiótica de los códigos», cuyos límites, derivados de asunciones ingenuamente metafísicas, se han puesto ya de manifiesto (Bonfantini, 1981); sino sobre todo porque me parece que el programa de investigación que se apunta en ellos es radicalmente inadecuado. La verdad es que calificar de «investigación» el programa de trabajo indicado por Hjelmslev es ya conceder demasiado: Porque en ciencia y en la reflexión epistemológica por «investigación» se entiende un proceso orientado a la innovación y al descubrimiento, no a la tautología y a la confirmación repetitiva. Se elaboran teorias para explicar "hechos sorprendentes" (para decirlo en términos de Peirce), y para abrir nuevas prospectivas sobre otros hechos conocidos y prever modalidades de ocurrencia de otra manera inobservables, que vendrán a ser luego, de un modo u otro, y abriendo así nuevos procesos, verificadas. En cambio, Hjelmslev nos dice tranquilamente que no quiere avanzar «un sistema de hipótesis», que por "teoría» él entiende una ordenada exposición de "ciertas premisas que el autor de la teoría sabe, en base a la experiencia precedente, que satisfacen las condiciones de aplicación a ciertos datos empíricos" (Hjelmslev, 1943: 16-17). Hjelmslev elimina de su horizonte todo "hecho sorprendente", por ejemplo el que en el lenguaje se entrecrucen innovación y conservación, constancia y cambio; se encuentra únicamente ceñido a la "constancia» de "objetos de un tipo específico"; ve que "se pueden describir de forma coherente"; piensa que hacerlo acarrea "una comprensión del objeto examinado" (Ibídem, 18-19). EI trabajo del teórico, según Hjelmslev, es simple y cómodo: "Se descubren ciertas 
propiedades presentes en ciertos objetos que la gente está de acuerdo en llamar lengua, y después se generalizan tales propiedades, dándolas por definición. A partir de este momento el teórico del lenguaje ha decidido ya, de forma arbitraria pero apropiada, a qué objetos podrá o no podrá aplicar su teoria. Después constituye, para todos los objetos del tipo especificado en la definición, un cálculo general en el que están previstos todos los casos concebibles» (Ibídem, 21).

En este punto, la consecuencia es evidente: si he escogido de forma suficientemente general (y genérica) las «propiedades presentes en ciertos objetos que la gente está de acuerdo en llamar lengua», es muy fácil que vuelva a encontrar estas mismas propiedades en los textos de todas las lenguas y de los sistemas de signifiación-comunicación con los que tropiece. Si en ellos falta algunas de dichas propiedades, puedo rearticular ocasionalmente la clasificación sin cambiar el principio clasificatorio.

Naturalmente, se trata pues de un trabajo de taxonomía, que podria resultar más o menos útil según los fines específicos de la investigación. Se dirá que también poner en orden el "sentido común" y el "saber común", inventariar, en suma, las «expectativas recurrentes» más generales y constantes del uso, el juego más regular y uniforme de los reenvíos en la superficie de los fenómenos lingüísticos, encierra una previsible utilidad.

Sí, pero para ir más allá de este trabajo de generalización empírica y de taxonomía, es necesario reconocerlo como tal y en sus propios límites. Por el contrario, diríase que Hjelmslev parece un Linneo que se creyera, confusamente, un Darwin.

A pesar de todo ello, es tal la azarosa imprevisibilidad de la generación del sentido, paradójicamente exorcizada precisamente por él, que a Hjelmslev le ha caído en suerte el haber dado un paso que habría de llevar a nuevos módulos interpretativos, más ricos y ágiles, en la semiótica.

Dicho paso consiste en haber sugerido una redefinición de la connotación que se revelaría notablemente fecunda, desencadenando un interesante proceso. A esta feliz jugada de Hjelmslev, fuera ya de esa crítica general sólo esbozada aquí sumariamente, está dedicado cuanto sigue.

II. Verdad es que a una persona que ha dedicado a la connotación un libro de muy extensa información e intensa inteligencia, me refiero a Catherine KerbratOrecchioni, le resulta una actitud científicamente injustificada el que «Eco, Ducrot, Greimas, Barthes y muchos otros más» hayan partido "piadosamente» del "más célebre esquema de 'décrochement' connotativo, tal y como lo ha formulado Hjelmslev" (1977: 80). Este esquema le parece, en efecto, demasiado abstracto y genérico para dar cuenta de la especificidad y variedad de los «hechos connotativos" (ibidem: 79 y passim). La solución a la que tiende $\mathrm{C}$. Kerbrat-Orecchioni es la de que a los significados connotativos les debe corresponder sobre el plano de la expresión unos "connotadores» con función paralela y no dependiente respecto a la expresión denotativa (ibídem: 12 ss., 85 ss., y passim).

$Y$ en efecto, a primera vista, la definición hjelmsleviana de la connotación como contenido de las "semióticas cuyo plano de la expresión es una semiótica" (1943: 122), puede parecer incongruente respecto a la experiencia y a la definición corriente y especializada de la connotación. Por connotación suele entenderse las 
informaciones de más, «subsidiarias» respeto a las informaciones primarias o necesarias en el reenvio denotativo (C. Kerbrat-Orecchioni, 1977: 15). El significado connotativo aparece así como "subjetivo», "sugestivo", «implícito", «emocional» (ibidem: $14,16,17,89-91$, y passim). ¿Y qué tiene que ver este significado 'lateral' con el significado denotativo?

Pues bien: la feliz jugada de Hjelmslev ha consistido precisamente en refutar esta aparente y apariencial 'lateralidad' por considerarla, digamos interpretándole, mero efecto de superficie de una relación más profunda, según la cual el significado connotativo está constituido mediante la denotación. En qué medida haya tenido Hjelmslev presentes las sugerencias de la tradición filosófica, que desde Occam a James Mill habian insistido en el fenómeno de la significación indirecta; y/o haya acogido ciertas tendencias del uso, para las que /connotar/vale también por «implicar tal o cual consecuencia o condición", en casi todas las lenguas europeas; y/o haya seguido los principios de «economia» propios de su metódica —todo ello no tiene mucha importancia. El hecho es que suministró un criterio para redefinir y reinterpretar la connotación como contenido de la denotación.

Como ha sido subrayado por Kerbrat-Orecchioni, en los Fundamentos Hjelmslev no se refiere nunca, hablando de la connotación, a «hechos precisos y localizados" (1977: 81). Queda así muy oscuro qué debe hacer operativamente el analista, con una definición que consiste meramente en la indicación de una relación entre dos (conjuntos de) entidades no bien descritas y apenas tipificadas. A pesar de todo, Hjelmslev consiguió abrir un proceso interpretativo importante a partir de la siguiente prescripción: «Una vez completo ya el análisis de la semiótica denotativa, se debe someter la semiótica connotativa a un análisis según el mismo procedimiento" (1943: 127). Aquellos que lo siguieron reinterpretaron esta prescripción en el sentido de que para considerar la connotación se requiere pasar a través del significado primero, o denotación, y añadir una especie de segundo movimiento de reenvío a ese primer movimiento que asocia el significante al significado inmediato.

III. Esta idea del 'doble movimiento' comienza a arraigar tal vez a partir de la representación icónico-diagramática que, como ahora recuerda exactamente Eco, fue primero "divulgada por Barthes" (Eco, 1984: 30):

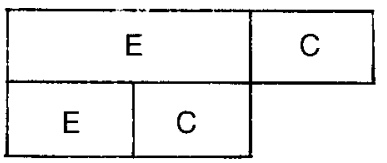

De aquí, de esta figura, se induce a poner espontáneamente entre paréntesis el 'comando' de la metáfora fisicista de la connotación "contenida». Se ve, en efecto, un espacio delimitado que sobresale de, y que está separado como otro, del espacio en el que se consuma el enlace primario entre «E» $y$ « $C$ ».

De ahi, probablemente, el proceso analítico seguido por Eco en su conocido ejemplo de la "bacia hídrica» (1968 y 1975). Tenemos aquí un caso, dice justamente Catherine Kerbrat-Orecchioni, de "cadena" o "cascada" de "asociaciones" (1977: 82-83).

En base a instrucciones codificadas, un técnico sabe que una cierta señal eléc- 
trica expresa el contenido «agua al nivel X». Hecha presente así la denotación, el técnico puede (el subrayado es siempre nuestro, no de Eco), con una segunda operación interpretativa, y sobre la base de su propio saber o bien de otras instrucciones, comprender que "agua al nivel $x$ " connota "peligro". $Y$ en ese momento puede decidir que «este peligro" comporta la obligación de "abrir las compuertas del dique».

En esta re-exposición mía se sugieren dos consideraciones: (1) que el ejercicio de interpretación connotativa no puede más que concernir a una ocurrencia sígnica, una ocurrencia de comunicación, y no a un término aislado en abstracto del fluir de la comunicación; (II) que en dicho ejercicio se pasa a través de al menos dos pasos interpretativos: el primero es obligatorio para la consideración de la ocurrencia sígnica misma como significante; mientras que el segundo se requiere para la comprensión del signo en cuanto inserto en un texto, en un contexto, y en un juego comunicativo (Bonfantini, 1984: 358; cf. Schmidt, 1973).

IV. Eco, proponiendo el ejemplo de análisis, no llegaba hasta estas consideraciones. Sin embargo, contemplando la forma de aquel mismo ejemplo suyo, no podia dejar de desplazar la atención desde el modelo estático de las "semióticas" de Hjelmslev al modelo procesual de la «semiosis»; y, recordando entonces la concepción peirceana de la significación, se encontraba ya en posición de introducir (con mayor relieve a partir de 1971) la categoría cardinal de la semiótica de Peirce: el interpretante.

Con ello, Eco contribuía a difundir entre los estudiosos la exigencia de una profundización en la prospectiva peirceana, tematizada ya como semiótica de la interpretación.

Hoy, a aquellos de nosotros que hemos recorrido un cierto trecho de este camino de profundización, nos resulta muy claro que la generación y la comunicación de significancia poseen los caracteres constitutivos e interrelacionados de la procesualidad, de la subjetividad, y de la inferencialidad.

V. Una vez captado y retenido que significar-interpretar es poner en marcha un proceso subjetivo-inferencial, denotación y connotación no parecerán ya lo que parecían en la «semiótica de los códigos»: no parecerán ya esos objetos cuyo estatus ontológico era de una bizarra y arbitraria metafísica - curiosas posibilidades antecedentes, en ese caso, las de la semiosis en carne y hueso, en acto, ésas con las que nos vamos a encontrar siempre dentro de las semiosis (no se sabe exactamente dónde), para remitirlas después, intactas, a su cajón arcano, o sea al Gran Libro de Dios. Denotación y connotación aparecerán en cambio como los términos de unas operaciones semióticas determinadas y formalmente determinables.

Entonces, denotación y connotación se distinguen al distinguir las operaciones semióticas de las que han resultado, y se distinguen a su vez en las posiciones relativas que alcanzan estas operaciones semiósicas en el flujo de la semiosis.

No hay por consiguiente términos que en si sean denotativos ni connotativos, no hay denotadores ni connotadores, sino solamente semiosis que pasan y 'se posan' 
en ciertos estadios. Por lo tanto, y según los puntos de partida y los contextos y juegos comunicativos, un interpretante que es interpretable según un mismo type (por ejemplo, el semema «león»), podrá verificarse como interpretante inmediato (o denotación, según la terminología de origen hjelmsleviano), como interpretante u/terior solicitado (o connotación), o como interpretante evocado dentro de la libertad de la semiosis ilimitada.

VI. Veamos con mayor análisis la forma general del proceso de interpretación.

PRIMER MOMENTO DEL PROCESO: Individuación de un acontecimiento como ocurrencia sígnica, y con ello, (re)presentación de la significancia como (re)clamo de sentido por medio, del movimiento inferencial (abductivo) del representamen sobre el objeto inmediato, a través del interpretante inmediato; en este primer momento del proceso, se pasa del significante al significado, constituyendo el tema para la semiosis posterior, que se desarrolla como sucesión de interpretantes y, por lo tanto, por pasos de significado a significado.

SEGUNDO MOMENTO DEL PROCESO: Serie de pasos inferenciales que conduce a la interpretación solicitada o exigida por el proceso pragmático que reconozco en el texto.

TERCER MOMENTO DEL PROCESO: Serie, en principio ilimitada, de pasos interpretativos, en la que el intérprete puede apartarse del programa pragmático del texto, procediendo por evocación, según una inferencialidad abductiva que, acentuando los "desplazamientos" (Ponzio, 1982) va más allá, en poiesis, de un orden (narración) prescrito.

Será mejor ahora ver un poco cómo funcionan estos principios frente a problemas algo más específicos. Por ejemplo, frente al problema de dar cuenta de esa clase de fenómenos connotativos que podemos agrupar bajo la categoría de implicito pragmático.

\section{B) La abducción en la connotación del implícito pragmático}

1. Según la propuesta lanzada por mí por primera vez en 1982, entre la dimensión inmediata, ilocutoria, del acto lingüístico, y la dimensión final, perlocutoria, se da la dimensión de la valencia efectivamente ocurrente del acto lingüístico, dentro de la duración propia del discurso y de la comunicación: dimensión que podemos llamar dialocutoria.

Para explicarme con un ejemplo: «Esta sopa está sosa» es, en su aspecto ilocutorio, obviamente un acto representativo; la finalidad última para la que dicho acto ha sido ejecutado por el emisor podrá ser, desde el punto de vista del intérprete, una cuestión bastante hipotética y de difícil elucidación; pero la valencia efectiva de "Esta sopa está sosa" está en su aspecto dialocutorio (en la valencia que esta aserción asume en un contexto y una circunstancia dados). Así, precedido de una secuencia de actos ilocutorios y preparado por un cierto sistema de figuras de comunicación, "Esta sopa está sosa» vendrá entendido como una petición elíptica de añadir sal a la sopa; precedido de otra secuencia de actos ilocutorios y preparado por otro sistema de figuras de comunicación, “Esta sopa está sosa» deberá ser 
en cambio interpretado como el veredicto negativo de un juez (en una competición de cocineros).

Según una terminología inspirada en Peirce, puede decirse que: el aspecto ilocutorio es el interpretante pragmático inmediato de un acto lingüístico, el aspecto dialocutorio el interpretante pragmático dinámico, y el aspecto perlocutorio el interpretante pragmático final. Pero puede también decirse que: el aspecto dialocutorio del discurso es el aspecto elemental, el aspecto dialocutorio el aspecto estructural, y el aspecto perlocutorio el aspecto estratégico-ermenéutico.

En este apartado se intentará mostrar cómo esta tripartición del sentido pragmático, combinándose con nuestra teoría de tipos de abducción, suministra el cuadro conceptual adecuado para la interpretación de los distintos géneros de implicitud (presuposición, sobreentendido, alusión) afrontados diversamente por Ducrot y otros autores.

II. Me sirvo ahora de tres advertencias o aclaraciones preliminares, que no voy a sostener con argumentaciones: se me ocurren entonces bajo los siguientes lemas.

PRIMER LEMA: DEFINICIÓN DE IMPLÍCITO. Implícito=sentido no explicitado ni expuesto por enumeración o listado por parte del emisor, sino interpretable a partir del texto e interpretándose para la comprensión del mismo.

SEGUNDO LEMA: CONVENCIONALIDAD DEL ASPECTO ILOCUTORIO. EI aspecto ilocutorio de un acto lingüístico cualquiera se reconoce por convención establecida. De esta convencionalidad del aspecto ilocutorio depende su inmediata descodificabilidad, por parte del destinatario o de cualquier oyente o lector, y por ende por parte del analista, sobre la sola base de la competencia lingüística (de lengua y de lenguaje).

TERCER LEMA: SENTIDO DEL ASPECTO DIALOCUTORIO. EI nivel dialocutorio es el nivel de sentido del acto lingüístico o, más en general, del texto, que responde a la siguiente pregunta del destinatario: «¿qué quiere de mí?». La forma de la respuesta puede expresarse icónicamente con la REPRESENTACIÓN siguiente:

\section{E (emisor)}

\section{REPRESENTACIÓN DE LA FORMA DEL SENTIDO DIALOCUTORIO}

\section{autorizándose}

al ejercicio del sentido y de

la influencia sobre mí, destinatario, mediante su constitución en

FCE (figura cualificada de enunciador), por vía de

a) presuposición en el juego comunicativo

o bien

b) alusión

o bien

c) declaración 
QUIERE

HE (hacer emprender)
HD (hacer desear o hacer creer precisamente que lo es) o bien

$\mathrm{HH}$ (hacer hacer)

bien

HS (hacer saber)

$X$ (el determinado hecho enunciado de tal y tal modo, o la determinada acción enunciada de tal y tal modo)

por mí, D (destinatario)

apelando a mí en cuanto dispuesto a identificarme con la

FAE (figura adecuada de enunciatario)

constituida por vía de

a) presuposición en el juego comunicativo

o bien

b) alusión

o bien

c) declaración

III. Resultará ahora comprensible el siguiente cuadro de distribución de lo implícito o la siguiente

REPRESENTACION TOPOLOGICA DE LO IMPLICITO

\begin{tabular}{|c|c|c|c|c|}
\hline & & \multicolumn{3}{|c|}{$S E N T I D O$} \\
\hline$A$ & ilocutorio & $\begin{array}{l}\text { VEHICULADO } \\
\text { COMO } \\
\text { PRESUPUESTO }\end{array}$ & MANIFIESTO & ENCONTRADO \\
\hline$S$ & dialocutorio & COMPORTADO & $\begin{array}{l}E \text { sobreen- } \\
V \text { tendido } \\
O \\
C \text { sugerido } \\
A \\
D \text { aludido } \\
O\end{array}$ & INDUCIDO \\
\hline$T$ & perlocutorio & $D E L A T A D O$ & ESCONDIDO & ADIVINADO \\
\hline & & $\begin{array}{l}\text { por } \\
\text { el acto }\end{array}$ & $\begin{array}{c}\text { por } \\
\text { el emisor }\end{array}$ & $\begin{array}{c}\text { por } \\
\text { el destinatario }\end{array}$ \\
\hline
\end{tabular}

IV. La tabla puede leerse y 'contarse' de forma socorrida, del siguiente modo: El sentido implícito del aspecto ilocutorio de un acto lingüístico está vehiculado por el acto mismo y, por consiguiente manifiesto — para y tenido por descubierto- por 
el propio emisor, y cómodamente localizable por el destinatario en su reserva de interpretaciones codificadas y habituales; por su parte, el sentido implicito del aspecto dialocutorio está 'comportado' o 'implicado' por el acto mismo, según reenvíos más o menos usuales pero, en cualquier caso, obvios por definición (de otra forma la comunicación carecería de efectividad), y por consiguiente evocado por el emisor (sobreentendido, sugerido, o aludido, según el carácter más o menos institucionalizado del frame en el que el acto ocurre, y según las estrategias específicas del emisor), e inducido por el destinatario con un poco de atención; en fin, el sentido implícito del aspecto perlocutorio, el "objetivo", está parcialmente delatado por el acto mismo, y por consiguiente, aunque escondido por definición por el emisor, se ofrece a las tentativas de adivinación del destinatario en base a sus indicios.

Si retomamos ahora el ejemplo de la «Sopa", nos percatamos claramente del carácter ascendente de la escala, en cuanto a dificultad e inventiva de hipótesis interpretativas, que aparece sintéticamente indicada por los tres adjetivos «encontrado», «inducido», "adivinado», de la última columna de la tabla. Pero para una comprensión más precisa debemos recurrir en este punto a nuestra teoría de tipos de abducción.

V. Por abducción, sabemos que Peirce entiende un argumento que obedece al esquema del siguiente ejemplo:

Estas judias son blancas

(pero) todas las judías de aquel saco son blancas

(luego) estas judias provienen de aquel saco (quizás)

RESULTADO

REGLA

CASO

Para una representación icónica de la abducción, que la libere de la complejidad de la generalización o cuantificación universal (v. Bonfantini, 1983), resulta útil emplear aquí también un simbolismo que retoma ciertos procedimientos de Casari (1959).

Tendremos entonces la siguiente representación de la abducción:

0

$\mathrm{H} \rightarrow \theta$

$H$

V1. Es fácilmente intuible de inmediato, y está ampliamente justificado en otro lugar (Bonfantini-Proni, 1980), que la "oriencia creativa» de la abducción, es decir su creatividad y originalidad, depende de la clase de ley-mediación a que se recurra. Tendremos entonces, volviendo sobre ello, tres grados o tipos de abducción:

PRIMER TIPO DE ABDUCCIÓN: La ley-mediación a la que recurrir para inferir el caso a partir del resultado nos viene dada de forma obligatoria y automática o semiautomática;

SEGUNDO TIPO DE ABDUCCIÓN: La ley-mediación a la que recurrir para inferir 
el caso a partir del resultado se adopta, por selección, de entre el ámbito de la enciclopedia disponible;

TERCER TIPO DE ABDUCCIÓN: La ley-mediación a la que recurrir para inferir el caso a partir del resultado está constituida ex novo, inventada.

Dentro del tercer tipo de abducción cabe distinguir tres subtipos, según la modalidad de constitución de la ley-mediación. Tendremos así:

PRIMER SUBTIPO: La ley-mediación es una mera extensión a otro campo semántico de una forma de implicación ya presente en la enciclopedia disponible;

SEGUNDO SUBTIPO: La ley-mediación conecta ex novo dos (conjuntos de) elementos ya presentes en el universo semántico de la enciclopedia disponible;

TERCER SUBTIPO: La ley-mediación introduce en su antecedente lógico un término ficticio.

Provistos de esta tipología, puede decirse ahora que en la comunicación cotidiana la comprensión del sentido del nivel ilocutorio reclama operaciones abductivas del primer tipo, la del nivel dialocutorio, del segundo tipo, y la del nivel perlocutorio combinaciones de abducción del segundo tipo y a veces también del tercer tipo.

VII. El sentido dialocutorio, en la medida en que es siempre, y por igual, sentido comportado, evocado, e inducido, requiere en el intérprete, para su comprensión, una forma constante de operaciones lógicas. Pero en la dialogicidad del juego comunicativo, el hecho de que el sentido evocado por el emisor esté sobreentendido, o bien sugerido, o bien, en fin, aludido, obedece a distintas estrategias y produce en el destinatario efectos de sentido diversos. El sobreentendido y la sugerencia parecen las estrategias normales durante los diálogos de rutina o los diálogos de solicitación corrientes (ver Bonfantini-Ponzio 1983 y 1986); le sirven al emisor para mantener fuera de discusión determinados lugares comunes reconocidos como tales. La alusión, en cambio, parece moverse en la indeterminación, la vaguedad, lo indefinido del sentido evocado. Dice Peirce (1931-1958: 5.447): «Un signo, objetivamente indeterminado respecto a algo, es objetivamente vago si y en cuanto le reserva las determinaciones ulteriores a otro signo concebible, sin señalar en todo caso al intérprete como delegado para dicho papel. Ejemplo: 'Alguien que podría mencionar me parece un poco presuntuoso'. Aquí se insinúa por alusión que la persona en cuestión es aquella a la que nos dirigimos. Pero el emisor no autoriza ni ésta interpretación ni ninguna otra aplicación de su enunciado. Puede inciuso muy bien, si quiere, decir que no da a entender con ello que sea la persona a la que se está dirigiendo".

En este caso de vaguedad y de alusión, tratado por Peirce, lo que (por mera conveniencia) permanece vago es sólo el referente del juicio, juicio que, en cambio, está plenamente determinado. Este tipo de alusión se configura así como un ardid, netamente agresivo, característico de un diálogo competitivo: consiste en hacer saber a! interlocutor que se posee una opinión negativa y ofensiva sobre él, tipificándola con precisión, pero tendiendo a paralizar toda posibilidad de respuesta mediante la ficción de la indeterminación del sujeto.

Pero hay otro tipo de vaguedad y alusión, que es el recíproco del tipo de la alusión agresiva: es la alusión cooperativa seductora. En este caso está determi- 
nado perfectamente el referente, y el interlocutor, y ningún otro, implicado al máximo; de dicho interlocutor se invoca una respuesta que queda, intencionalmente, plenamente abierta al juego de la comunicación, mediante la ambigüedad, la indefinición, o la pluralidad de sugerencias del sentido evocado y ofrecido al uhacer dialógico» del interlocutor.

(Traducido del italiano por Ángel Herrero)

\section{Referencias bibliográficas}

Austin, John Langshaw (1962), How to Do Things with Words, Londres, Oxford Univ. Press.

Barthes, Roland (1964), Elementi di semiologia, Turín, Einauidi, 1976 (9. $\left.{ }^{a}\right)$.

Bonfantini, Massimo Achille (1981), "Le tre tendenze semiotiche del Novecento», Versus, 30 (ahora también en 1984).

- (1982), L'occhio sull'argomentazione nella comunicazione di masa, en Rositi 1982 (ahora también en Bonfantini 1984).

- (1983), Abduction, A Priori, Brain: for a Research Program, en BonfantiniProni (comp.) 1983.

- (1984), Semiotica ai media, Bari, Adriatica.

Bonfantini, Massimo Achille-Proni, Giampaolo (1980), «To guess or not to guess?» en Scienze umane, 6 (ahora también en Eco-Sebeok (eds.) 1983.

Bonfantini, Massimo Achille-Proni, Augusto (comp.) (1983), "L'abduzione», Versus, 34.

Casari, Ettore (1959), Lineamenti di logica matematica, Milán, Feltrinelli.

Eco, Umberto (1968), La struttura assente. Milán, Bompiani.

- (1971), Le forme del contenuto, Milán, Bompiani.

- (1975), Trattato di semiotica generale. Milán, Bompiani.

- (1984), Semiotica e filosofia del linguaggio; Turín, Einauidi.

Eco, Umberto; Sebeok, Thomas A. (eds.) (1983), The Sign of Three, Bloomington, Indiana univ. press (en la trad. italiana del mismo año, II segno di tre, de Giampaolo Proni, Milán, Bompiani). 